\title{
ADMISSIBLE RELAXATION IN OPTIMAL CONTROL PROBLEMS FOR INFINITE DIMENSIONAL UNCERTAIN SYSTEMS ${ }^{1,2}$
}

\author{
N.U. AHMED \\ University of Ottawa \\ Department of Electrical Engineering, Department of Mathematics \\ and Department of Systems Science \\ Ottawa, Ontario, CANADA \\ X. XIANG \\ Guizhou University \\ Department of Mathematics \\ Guiyang, Guizhou \\ PEOPLE'S REPUBLIC OF CHINA
}

\begin{abstract}
In this paper we present a result on admissible relaxation for a class of systems governed by an uncertain evolution equation on Banach space. We show that the set of original trajectories is dense in the set of relaxed trajectories and that under certain assumptions the relaxed system is equivalent to the original system.
\end{abstract}

Key words: Uncertain evolution equation, strongly nonlinear, monotone operators, optimal controls, relaxed controls.

AMS (MOS) subject classifications: $\quad 93 \mathrm{C} 25,49 \mathrm{~A} 35,49 \mathrm{~B} 22$.

\section{BASIC NOTATIONS AND INTRODUCTION}

Let $H$ be a separable Hilbert space and $V$ a subspace of $H$ having the structure of a reflexive Banach space with the embedding $V \subset H$ being dense and compact. Identifying $H$ with its dual we have $V \subseteq H \subset V^{*}$ where $V^{*}$ is the topological dual of $V$. Let $\langle x, y\rangle$ denote the pairing of an element $x \in V$ and an element $y \in V^{*}$. If $x, y \in H$, then $\left.\langle x, y\rangle=x, y\right)$ where $(, \quad)$ is the scalar product in $H$. The norm in any Banach space $X$ will be denoted by $\|$ $\|_{X}$. Let $I=[0, T], 0<T<\infty$, and $p, q \geq 1$ such that $(1 / p)+(1 / q)=1$ and $2 \leq p<\infty$. Denote $L_{p}(V) \equiv L_{p}(I, V), L_{q}\left(V^{*}\right) \equiv L_{q}\left(I, V^{*}\right)$. For $p, q$ satisfying the preceding conditions it follows from the reflexivity of $V$ that both $L_{p}(V)$ and $L_{q}\left(V^{*}\right)$ are reflexive Banach spaces. The

${ }^{1}$ Received: March, 1992. Revised: August, 1992.

${ }^{2}$ This work is supported in part by the National Science and Engineering Research Council of Canada under grant no. A7190. 
pairing between $L_{p}(V)$ and $L_{q}\left(V^{*}\right)$ is denoted by $\ll$, $\gg$. Clearly, for $u, v \in L_{2}(H)$, $\ll u, v \gg=((u, v))$ is the scalar product in the Hilbert space $L_{2}(H)$. Define the set $W_{p, q}=\left\{y \in L_{p}(V): D y \equiv(d / d t) y \in L_{q}\left(V^{*}\right)\right\}$ where the derivative is understood in the sense of distributions. Furnished with the topology induced by the norm, $\|x\|_{p, q} \equiv\|x\|_{L_{p}(V)}+$ $\|D x\|_{L_{q}\left(V^{*}\right)}, W_{p, q}$ is a Banach space. Further it is well known that the embedding $W_{p, q}$ $\subset C(I, H)$ is continuous (see [1], Theorem 1.2.15) and that the injection $W_{p, q} \rightarrow L_{p}(H)$ is compact (see [11], p. 450; [12], Theorem 5.1 , p. 58).

In many engineering problems, a system may have only partial description in the sense that it may be governed by a differential equation containing many parameters or coefficients whose probability law is not accurately known except perhaps it's support. Thus an uncertain system may be described by the following evolution equation:

$$
\begin{gathered}
(d / d t) x(t)+A(t, x(t))=\int_{\Gamma} g(t, x(t), \omega) \mu(\omega)+B(t, u(t)) \\
x(0)=x_{0}, \mu \in \mathcal{H}^{+}+(\Gamma), u \in \mathcal{U}_{a d},
\end{gathered}
$$

where the perturbing operator $g$ contains the unknown parameters which take values from the set $\Gamma$, but neither the values of the parameter nor the underlying probability law $\mu$ is know. Let $\mathfrak{H}^{+}(\Gamma)$ denote the space of probability measures on the Borel subsets of $\Gamma$ where $\Gamma$ is any compact metric space. Clearly the underlying probability measure must be an element of this space. The set $\mathcal{u}_{a d}$ represents the class of admissible (original) controls to be defined shortly. For a given $u \in \mathcal{U}_{a d}$ and $\mu \in \mathcal{H}^{+}(\Gamma)$, let $x(\mu, u)$ denote the solution of the evolution equation (1) and

$$
J(\mu, u) \equiv \int_{I} L(t, M x(\mu, u), u) d t
$$

the corresponding cost functional. Since the measure $\mu$ is unknown to the analyst, he takes a pessimistic view and tries to minimize the maximum possible cost. For the fixed control $u$, define

$$
J_{0}(u) \equiv \sup \left\{J(\mu, u) \equiv \int_{I} L(t, M x(\mu, u), u) d t, \mu \in \mathcal{H}^{+}(\Gamma)\right\}
$$

In other words, $J_{0}$ represents the maximum risk associated with the control $u$. In [6], we considered the following optimal control problem for this system: find $u^{0} \in \Psi_{a d}$, such that

$$
J_{0}\left(u^{0}\right)=\iota n f\left\{J_{0}(u), u \in \mathcal{U}_{a d}\right\}
$$


We shall call this optimal control problem the (original) problem $\left(P_{0}\right)$. Later we introduce the relaxed control problem $\left(P_{r}\right)$.

For convenience of presentation we shall often write the differential equation (1) as a functional equation,

$$
D x+A(x)=\widehat{g}(x, \mu)+B(u),
$$

with the equality understood in the sense of equivalence in $L_{q}\left(V^{*}\right)$. Here, we have used $\widehat{g}(\xi, \nu) \equiv \int_{\Gamma} g(., \xi, \alpha) \nu(d \alpha)$ denote the function

$$
t \rightarrow \int_{\Gamma} g(t, \xi, \alpha) \nu(d \alpha) \text { from } I \text { to } V^{*} \text { for } \nu \in \mathscr{H}^{+}(\Gamma) \text { and } \xi \in H .
$$

The system (1) represents a large class of uncertain evolution equations with the uncertainty originating from the lack of knowledge of the probability law $\mu$. Hence the designer wishes to find a control policy to minimize the maximum risk.

As stated above, in [6] we proved an existence theorem for optimal original controls. In this paper we present a result on admissible relaxation. In control theory it is well known that in the absence of convexity, optimal controls may not exist even for finite dimensional systems. However, under certain assumptions, the convexified problem may have an optimal relaxed control. The question then arises as to if the relaxed optimal solution can be approximated to any degree of accuracy by a solution of the original problem. If this can be done then for all practical purposes the relaxation is admissible. In other words, 6 -optimal controls can be found from the original (physically realizable) controls. We go beyond that and show that under reasonable hypotheses the relaxed system is equivalent to the original one. For finite dimensional systems similar questions have been considered by Clarke [7].

\section{BASIC ASSUMPTIONS}

We prove our results under the following basic assumptions:

(A) $\quad A: I \times V \rightarrow V^{*}$ is a map so that

(1) $t \rightarrow A(t, x)$ is measurable,

(2) $x \rightarrow A(t, x)$ is monotone and hemicontinuous, that is, $\langle A(t, x)-A(t, y), x-y\rangle \geq 0$, $A(t, x+\sigma y) \stackrel{w}{\rightarrow} A(t, x)$ in $V^{*}$ as $\sigma \rightarrow 0$ for all $x, y \in V$,

(3) there exist positive constants $c_{1}, c_{2}, c_{3}$ such that

$$
\|A(t, x)\|_{V^{*}} \leq c_{1}\left(1+\|x\| p^{-1}\right) \text { for } x \in V
$$


and

$$
\langle A(t, x), x\rangle+c_{3}\|x\|_{H}^{2} \geq c_{2}\|x\| P \text { for } x \in V .
$$

$(G): \quad g: I \times V \times \Gamma \rightarrow H$ satisfying

(1) $t \rightarrow g(t, x, \omega)$ is a measurable function,

(2) $x \rightarrow g(t, x, \omega)$ is both continuous and weakly continuous,

(3) there exist positive numbers $\alpha, \beta, \gamma$ such that for all $(t, x) \in I \times V$ $(g(t, x, \omega), x) \leq \gamma, \quad$ and $\quad\|g(t, x, \omega)\|_{H} \leq \alpha\|x\| p^{-1}+\beta \quad$ for $\quad$ all $(t, x, \omega) \in I \times V \times \Gamma$,

(4) $\omega \rightarrow g(t, x, \omega)$ is continuous from $\Gamma$ to $H_{w} \equiv\left(H, \tau_{w}\right)$ uniformly on $I \times V$.

$(U): \quad U: I \rightarrow c c(Y)=$ (the family of nonempty closed convex subsets of $Y$ ) is a measurable multifunction satisfying $U(t) \subseteq \mathcal{U}$ for almost all $t \in I$ where $\mathcal{U}$ is a fixed weakly compact convex subset of a separable reflexive Banach space $Y$. For the admissible controls we choose the set

$$
\left\{u_{a d} \equiv u \in L_{\infty}(Y): u(t) \in U(t) \text { a.e. }\right\} \text {. }
$$

$(B): \quad B: I \times \mathcal{u} \rightarrow H$ is measurable in $t$ and sequentially continuous in $u$ with respect to the weak topologies in $\mathcal{U}$ and $H$ satisfying $\int_{I}\|B(t, \sigma)\| q_{H} d t<\infty$ for $\sigma \in \mathcal{U}$ and $1<q \leq 2$.

(L): Let $E$ be a separable Banach space and $L: I \times E \times Y \rightarrow \bar{R} \equiv R \cup\{\infty\}$ be a mapping satisfying

(1): $\quad(t, e, y) \rightarrow L(t, e, y)$ is Borel measurable and bounded on bounded subsets of $I \times E \times Y$,

(2): $y \rightarrow L(t, e, y)$ is continuous for all $(t, e) \in I \times E$,

(3): $e \rightarrow L(t, e, y)$ is continuous for all $(t, y) \in I \times \mathcal{U}$,

(4): $\varphi(t)-\lambda\left(\|e\|_{E}+\|y\|_{Y}\right) \leq L(t, e, y)$ a.e. with $\varphi \in L_{1}, \lambda \geq 0$.

$(M): M: L_{p}(V) \rightarrow L_{p}(E)$ is an operator so that, for every sequence $\left\{x_{n}\right\} \subseteq W_{p, q}$ weakly convergent to $x \in W_{p, q}$, the sequence $\left\{M x_{n}\right\}$ has a subsequence that converges strongly to $M x$ in $L_{p}(E)$.

Remark: Note that the perturbing operator $g$ is not assumed to be monotone.

\section{MAIN RESULTS}

Lemma A: Suppose the assumptions $(A),(G),(B),(U),(L)$ and $(M)$ hold. Then for each fixed $u \in \mathcal{U}_{a d}$, there exists a measure $\mu^{0} \in \mathcal{H}^{+}(\Gamma)$ such that 


$$
J_{0}(u) \equiv \sup \left\{J(\mu, u): \mu \in \mathfrak{H}^{+}(\gamma)\right\}=J\left(\mu^{0}, u\right)
$$

and $x^{0}=x\left(\mu^{0}, u\right)$ is a weak solution of the evolution equation (1) corresponding to the pair $\left\{\mu^{0}, u\right\}$.

Proof: For fixed $u \in \mathcal{u}_{a d}$, define $J_{0}(u) \equiv \sup \left\{J(\mu, u), \mu \in \mathcal{H}^{+}(\Gamma)\right\} . \quad$ By assumption (L1), $J_{0}(u)$ is finite. Then there exists a sequence $\mu^{n} \in \mathcal{H}^{+}(\Gamma)$ and a solution $x^{n} \equiv x\left(\mu^{n}, u\right)$ of the system (1) such that $J\left(\mu^{n}, u\right) \rightarrow J_{0}(u)$ as $n \rightarrow \infty$. Since $\mathcal{H}^{+}(\Gamma)$ is compact in the $w^{*}$-topology (see [9]) there exists a subsequence of the sequence $\left\{\mu^{n}\right\}$, relabeled as $\left\{\mu^{n}\right\}$, and a $\mu^{0} \in \mathcal{H}^{+}(\Gamma)$ such that $\mu^{n} \stackrel{w^{*}}{\rightarrow} \mu^{0}$. By virtue of corollary 2 of [6] there exists an $x^{0} \in W_{p, q}$ such that $x^{n} \stackrel{w}{\rightarrow} x^{0}$ in $W_{p, q}$ and $x^{n} \stackrel{s}{\rightarrow} x^{0}$ in $L_{p}(H)$. First we prove that $x^{0}$ is a solution of equation (1) corresponding to the pair $\left\{\mu^{0}, u\right\}$ and then we show that $J_{0}(u)=J\left(\mu^{0}, u\right)$. Clearly

$$
D x^{n}+A\left(x^{n}\right)=\widehat{g}\left(x^{n}, \mu^{n}\right)+B(u)
$$

and hence, scalar multiplying this by $x^{n}-x^{0}$, we obtain

$$
\ll D x^{n}, x^{n}-x^{0} \gg+\ll A\left(x^{n}\right), x^{n}-x^{0} \gg=\ll \widehat{g}\left(x^{n}, \mu^{n}\right), x^{n}-x^{0} \gg+\ll B(u), x^{n}-x^{0} \gg
$$

which can be written as

$$
\begin{gathered}
\ll D x^{n}, x^{n}-x^{0} \gg+\ll A\left(x^{n}\right)-\widehat{g}\left(x^{n}, \mu^{0}\right), x^{n}-x^{0} \gg \\
=\ll \widehat{g}\left(x^{n}, \mu^{n}\right)-\widehat{g}\left(x^{n}, \mu^{0}\right), x^{n}-x^{0} \gg+\ll B(u), x^{n}-x^{0} \gg .
\end{gathered}
$$

From the convergence results given above, it is easy to verify that

$$
\ll D x^{n}, x^{n}-x^{0} \gg=(1 / 2)\left\|x^{n}(T)-x^{0}(T)\right\|_{H}+\ll D x^{0}, x^{n}-x^{0} \gg \rightarrow 0 \text { as } n \rightarrow \infty
$$

and

$$
\ll B(u), x^{n}-x^{0} \gg=\left(\left(B(u), x^{n}-x^{0}\right)\right) \rightarrow 0 \text { as } n \rightarrow \infty .
$$

Clearly, by assumption (G3), we have

$$
\begin{gathered}
\left|\ll \widehat{g}\left(x^{n}, \mu^{n}\right)-\widehat{g}\left(x^{n}, \mu^{0}\right), x^{n}-x^{0} \gg\right| \\
\leq 2\left\{\alpha\left(\left\|x^{n}\right\|_{L_{p}(V)}\right)^{(p / q)}+\beta(T)^{(1 / q)}\right\}\left\|x^{n}-x^{0}\right\|_{L_{p}(H)} .
\end{gathered}
$$

Since $\left\{x^{n}\right\}$ is contained in a bounded subset of $W_{p, q}$ and $x^{n} \stackrel{s}{\rightarrow} x^{0}$ in $L_{p}(H)$, it follows from this that

$$
\ll \widehat{g}\left(x^{n}, \mu^{n}\right)-\widehat{g}\left(x^{n}, \mu^{0}\right), x^{n}-x^{0} \gg \rightarrow 0 \text { as } n \rightarrow \infty
$$


Then it follows from (4)-(7) that

$$
\ll A\left(x^{n}\right)-\widehat{g}\left(x^{n}, \mu^{0}\right), x^{n}-x^{0} \gg \rightarrow 0 \text { as } n \rightarrow \infty .
$$

Thus by Proposition 2 of [5], we conclude that

$$
A\left(x^{n}\right)-\widehat{g}\left(x^{n}, \mu^{0}\right) \stackrel{w}{\rightarrow} A\left(x^{0}\right)-\widehat{g}\left(x^{0}, \mu^{0}\right) \text { in } L_{q}\left(V^{*}\right) .
$$

For any $\xi \in L_{p}(V)$ we write

$$
\begin{gathered}
\ll D x^{n}, \xi \gg+\ll A\left(x^{n}\right)-\widehat{g}\left(x^{n}, \mu^{0}\right), \xi \gg \\
=\ll \widehat{g}\left(x^{n}, \mu^{n}\right)-\widehat{g}\left(x^{n}, \mu^{0}\right), \xi \gg+\ll B(u), \xi \gg .
\end{gathered}
$$

Recall that

$$
\left.D x^{n} \stackrel{w}{\rightarrow} D x^{0} \text { in } L^{q}(V)^{*}\right)
$$

By virtue of assumption $(G 4)$ and the fact that $\mu^{n} \stackrel{w^{*}}{\rightarrow} \mu^{0}$ in $\mathcal{H}^{+}+(\Gamma)$, we have

$$
\ll \widehat{g}\left(x^{n}, \mu^{n}\right)-\widehat{g}\left(x^{n}, \mu^{0}\right), \xi \gg \rightarrow 0 \text { as } n \rightarrow \infty .
$$

Since $\xi \in L_{p}(V)$ is arbitrary, it follows from (8)-(11) that

$$
D x^{0}+A\left(x^{0}\right)=\widehat{g}\left(x^{0}, \mu^{0}\right)+B(u) \text { in } L_{q}\left(V^{*}\right) .
$$

Further it follows from the embedding $W_{p, q} \rightarrow C(I, H)$ and the fact that $x^{n}(0)=x_{0}$ for all $n$, that $x^{0}(0)=x_{0}$. Hence we conclude that $x^{0}=x\left(\mu^{0}, u\right)$, that is, $x^{0}$ is a weak solution of equation (1) corresponding to $\mu^{0}$ and $u$. By virtue of assumptions $(L 1, L 2, L 4)$ and $(M)$, one can easily prove that $J\left(\mu^{n}, u\right) \rightarrow J\left(\mu^{0}, u\right)$ as $n \rightarrow \infty$. Thus $J_{0}(u)=J\left(\mu^{0}, u\right)$ and this proves the Lemma.

Now we consider the relaxed problem $\left(P_{r}\right)$, associated to the original problem $\left(P_{0}\right)$, where the class of admissible controls $\mathcal{u}_{a d}$ is replaced by measure valued controls $u_{r} \equiv\left\{\eta: I \rightarrow M^{+}(น): \eta_{t}(U(t))=1\right.$ for all $\left.t \in I\right\}$. Note that, with respect to the weak topology, $\mathcal{U}$ is a compact polish space. We assume that $\mathcal{U}_{r}$ is furnished with the Young topology. Recall that $\eta^{n} \rightarrow \eta$ in the Young topology if for every $f \in L_{1}(I, C($ น $)) \equiv L_{1}(C($ (น))

$$
\int_{I \times \text { Q }} f(t, \sigma) \eta_{t}^{n}(d \sigma) d t \rightarrow \int_{I \times \mho} f(t, \sigma) \eta_{t}(d \sigma) d t .
$$

By Dunford-Pettis theorem, we may consider $L_{\infty}(I, M(\Psi)) \equiv L_{\infty}(M(\mathcal{Q}))$ to be the dual of $L_{1}(C(\mathcal{u}))$. Thus $u_{r} \subseteq L_{\infty}\left(M^{+}(\mathcal{u})\right) \subset L_{\infty}(M(\mathcal{u}))$. Note that by identifying each $u \in \mathcal{u}_{a d}$ with the corresponding Dirac measure $\delta_{u(\cdot)}$ we may embed $\mathcal{U}_{a d}$ in $\mathcal{U}_{r}$. For an excellent discussion of relaxed controls, see Warga [11, p. 263-]. 
The relaxed problem $\left(P_{r}\right)$ can then be described as follows: for each $\eta \in \mathcal{u}_{r}$ define

$$
J_{0}(\eta)=\sup \left\{\int_{I} \widehat{L}\left(t, M x(t, \mu, \eta), \eta_{t}\right) d t, \mu \in \mathscr{A}^{+}(\Sigma)\right\}
$$

where $\widehat{L}(t, z, \nu)=\int_{\mathcal{U}} L(t, z, \sigma) \nu(d \sigma)$ for $(t,, z, \nu) \in I \times E \times M^{+}(\mathcal{Q})$; and $x \equiv x(\cdot, \mu, \eta)$ is the solution of the relaxed evolution equation,

$$
\begin{gathered}
D x+A(t, x)=\widehat{g}(t, x, \mu)+\widehat{B}(t, \eta), t \in I, \\
x(0)=x_{0}, \mu \in \mathcal{H}^{+}(\Gamma), \eta \in \mathcal{U}_{r},
\end{gathered}
$$

corresponding to the parameter $\mu$ and the control measure $\eta$.

Here we have used the notations

$$
\widehat{g}(t, x, \mu) \equiv \int_{\Gamma} g(t, x, \alpha) \mu(d \alpha), \widehat{B}(t, \eta) \equiv \int_{\mathscr{U}} B(t, \sigma) \eta_{t}(d \sigma) .
$$

The problem is to find a control measure $\eta^{0} \in \mathcal{U}_{r}$ such that

$$
J_{0}\left(\eta^{0}\right)=\inf \left\{J_{0}(\eta), \eta \in u_{r}\right\}
$$

Clearly the control $\eta^{0}$ minimizes the maximum risk. This is the relaxed problem for the uncertain system (13).

The following relaxation theorem is the main result of this paper. Let $\chi_{0}$ denote the trajectories of the original control problem $P_{0}$ and $\chi_{r}$ denote the trajectories of the relaxed problem $P_{r}$.

Theorem B: Suppose the assumptions of Lemma A, including uniqueness of solutions of the evolution equations (1) (or equivalently (3)) and (13), hold. Then

(1): The set $\chi_{0}$ is dense in $\chi_{r}$.

(2): If the pair $\left\{x^{0}, u^{0}\right\} \in W_{p, q} \times \mathrm{U}_{a d}$ is optimal for the original problem $\left(P_{0}\right)$, then it is also optimal for the relaxed problem $\left(P_{r}\right)$.

Proof: (1): First we show that $\chi_{0}$ is dense in $\chi_{r}$. Let $y \in \chi_{r}$ be the solution of equation (13) corresponding to the relaxed control $\eta \in \mathcal{u}_{r}$ and a fixed but arbitrary $\mu^{0} \in \mathcal{H}^{+}(\Gamma)$. By virtue of Corollary 3 of Balder [8], there exists a sequence of ordinary

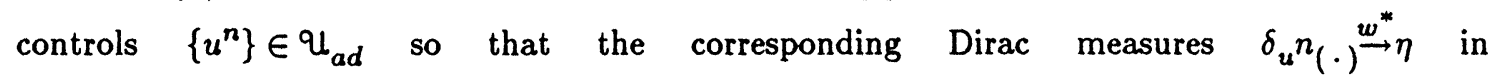
$L_{\infty}\left(M^{+}(\right.$$\left.)\right) \subset L_{\infty}\left(M(\right.$ U) $) \equiv\left(L_{1}(C(\mathcal{u}))\right)^{*}$. For the fixed $\mu^{0}$, consider the original system (1) corresponding to the sequence of ordinary controls $\left\{u^{n}\right\}$ and let $\left\{y^{n}\right\} \in \chi_{0}$ denote the 
corresponding solutions. By virtue of Lemma 1 of [6], we have $y^{n} \stackrel{w}{\rightarrow} x^{*}$ in $W_{p, q}$ and hence, by compactness of the embedding $W_{p, q} \rightarrow L_{p}(H), y^{n} \stackrel{s}{\rightarrow} x^{*}$ in $L_{p}(H)$. Since $u^{n}$ converges to $\eta$ in the Young topology, we have, for every $\xi \in L_{p}(H)$,

$$
\begin{gathered}
\ll B\left(u^{n}\right), \xi \gg \equiv \int_{I}\left(B\left(t, u^{n}(t)\right), \xi(t)\right)_{H} d t \rightarrow \int_{I \times \mho}(B(t, \sigma), \xi(t)) \eta_{t}(d \sigma) d t \\
\equiv \ll \widehat{B}(\eta), \xi \gg .
\end{gathered}
$$

Using this fact and following the same procedure as in Lemma $A$, one can easily verify that $x^{*}$ is a solution of equation (13) corresponding to $\mu^{0}$ and the relaxed control $\eta$ and that by virtue of uniqueness, $x^{*}=y$. This shows that, under our basic assumptions, any relaxed trajectory is the limit of a suitable sequence of original trajectories, there by proving that $\chi_{0}$ is dense in $\chi_{r}$.

(2): We prove this by establishing a contradiction. Suppose the assertion is false. Then there exists an $\eta^{0} \in \mathrm{u}_{r}$ and a $\delta>0$ such that

$$
J_{0}\left(\eta^{0}\right) \leq J_{0}\left(u^{0}\right)-\delta
$$

Since $U_{a d}$ is dense in $\varkappa_{r}$ in the Young topology, there exists a sequence of ordinary controls $\left\{u^{n}\right\}$ that converges in the Young topology to $\eta^{0}$. By virtue of Lemma $A$ there exists a sequence $\left\{\mu^{n}\right\} \in \mathcal{H}^{+}(\Gamma)$ corresponding to the sequence $\left\{u^{n}\right\}$ such that

$$
J_{0}\left(u^{n}\right)=J\left(\mu^{n}, u^{n}\right)=\sup \left\{J\left(\mu, u^{n}\right), \mu \in \mathscr{A}^{+}(\Gamma)\right\} .
$$

Let $x^{n} \equiv x\left(\mu^{n}, u^{n}\right)$ be the unique solution of the equation (1) corresponding to the pair $\left\{\mu^{n}, u^{n}\right\} \in \mathcal{H}^{+}(\Gamma) \times \mathcal{U}_{a d}$. Since the solutions lie in a bounded subset of $W_{p, q}$ (see Lemma 1 of [6]) and $\mathcal{H}^{+}(\Gamma)$ is compact, there exists a subsequence, relabeled as such, and $\left\{x^{*}, \mu^{*}, \eta^{0}\right\} \in W_{p, q} \times \mathcal{H}^{+}(\Gamma) \times \mathcal{u}_{r}$ such that

$$
\begin{gathered}
x^{n} \stackrel{⿱ w}{\rightarrow} x^{*} \text { in } W_{p, q}, \\
\mu^{n} \stackrel{w^{*}}{\rightarrow} \mu^{*} \text { in } \mathcal{H}^{+}(\Gamma), \\
u^{n} \stackrel{w^{*}}{\rightarrow} \eta^{0} \text { in } L_{\infty}\left(M^{+}(\mathcal{Q})\right) .
\end{gathered}
$$

Let $x\left(\mu^{*}, \eta^{0}\right)$ denote the unique solution of the relaxed evolution equation (13) corresponding to the pair $\left\{\mu^{*}, \eta^{0}\right\}$. Using (14) and repeating the arguments of Lemma $A$, one can easily justify that $x^{*}=x\left(\mu^{*}, \eta^{0}\right)$. Then by using the continuity assumption as given in (L2), and the assumptions $(L 3)$ and $(M)$ it follows from Young's topology that we have $\lim J_{0}\left(u^{n}\right)=J\left(\mu^{*}, \eta^{0}\right)$. Hence, for the given $\delta$ (see equation (15)), there exists an integer $n_{0}$ 
such that

$$
J_{0}\left(u^{n}\right) \leq J\left(\mu^{*}, \eta^{0}\right)+(\delta / 2) \text { for } n \geq n_{0} .
$$

Since, by definition, $J_{0}\left(\eta^{0}\right) \equiv \sup \left\{J\left(\mu, \eta^{0}\right), \mu \in \mathcal{A}^{+}(\Gamma)\right\}$ it is clear that

$$
J\left(\mu^{*}, \eta^{0}\right) \leq J_{0}\left(\eta^{0}\right)
$$

It follows from (15), (18) and (19) that

$$
J_{0}\left(u^{n} 0\right) \leq J_{0}\left(u^{0}\right)-(\delta / 2) .
$$

This contradicts the optimality of $u^{0}$ and hence the assertion of the theorem holds. This completes the proof.

Remark $C$ : A sufficient condition for uniqueness of solutions of the evolution equations (2) and (13) is that the operator $A$ be monotone and $g$ locally Lipschitz in $x$ on $H$.

Remark D: In this paper the duality $L_{\infty}(M($ น $))=\left(L_{1}(C(\text { น }))\right)^{*}$ has been referred to as Dunford-Pettis theorem. An anonymous reviewer of this paper pointed out the inappropriateness of this attribution and cited the authors Ionescu-Tulcea (see [13]) who gave the original proof of this duality.

\section{ACKNOWLEDGEMENT}

The authors would like to thank the anonymous reviewers of this paper for their valuable suggestions and comments.

\section{REFERENCES}

[1] N.U. Ahmed and K.L. Teo, “Optimal Control of Distributed Parameter Systems", North Holland, New York, Oxford, 1981.

[2] N.U. Ahmed, "Existence of optimal controls for a class systems governed by differential inclusions on a Banach space”, JOTA 50, 2, (1986), pp. 213-237.

[3] N.U. Ahmed, "Existence of optimal relaxed controls for differential inclusions on Banach space", Nonlinear Analysis and Applications, Lect. Notes in Pur 8 Applied Math, Marcel Dekker, Inc. Vol. 109 (ed. V. Lakshmikantham), (1987), pp. 39-49.

[4] N.U. Ahmed, "Optimization and identification of systems governed by evolutoin equations on Banach space", Pitman Res. Notes in Math. Ser., Vol. 184, Longman Scientific \& Technical, England and John Wiley \& Sons, New York, 1988.

[5] N. Hirano, "Nonlinear evolution equations with nonmonotone perturbations", Nonlinear Anal. T.M.A., 13, (1989), pp. 599-609. 
[6] N.U. Ahmed and X. Xiang, "Optimal control of infinite dimensional uncertain systems", (to appear).

[7] F. Clarke, "Admissible relaxation in variational and control problems", J. Math. Anal. Appl., 51, (1975), pp. 557-576.

[8] E. Balder, "A general denseness result for relaxed control theory", Bull. Austr. Math. Soc., 30, (1984), pp. 463-475.

[9] T. Parathasarathy, "Probabilities on Metric Spaces", Academic Press, New York and London, 1972.

[10] J. Warga, “Optimal Control of Differential and Functional Equations”, Academic Press, New York and London, 1972.

[11] E. Zeidler, “Nonlinear Functional Analysis and its Applications II", Springer, New York, 1990.

[12] J.L. Lions, “Quelques Methodes de Resolution des Problèmes aux Limites Non-Linéires”, Dunod, Paris, 1969.

[13] A. Ionescu and C. Tulcea, "Topics on the Theory of Lifting", Springer, Berlin, 1969. 


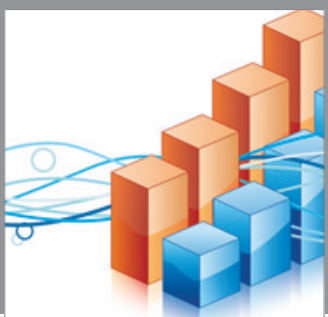

Advances in

Operations Research

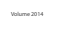

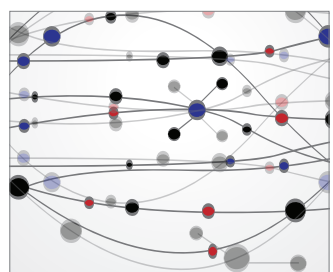

\section{The Scientific} World Journal
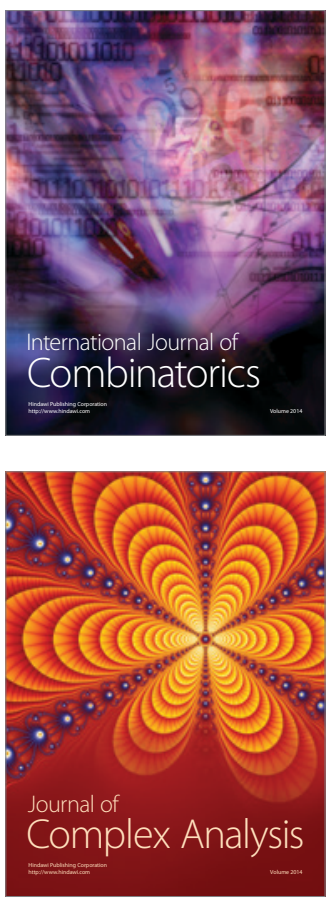

International Journal of

Mathematics and

Mathematical

Sciences
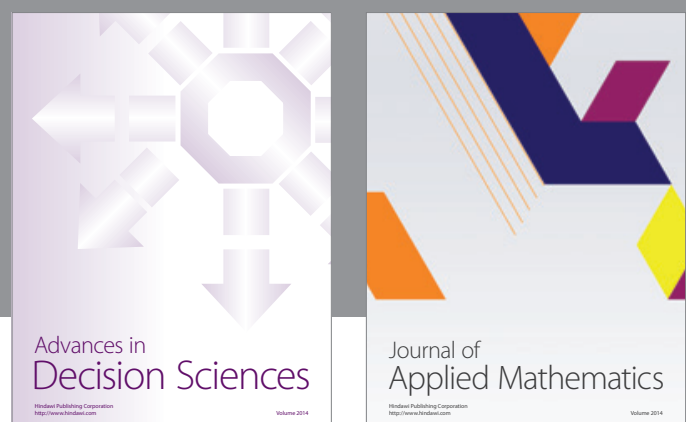

Journal of

Applied Mathematics
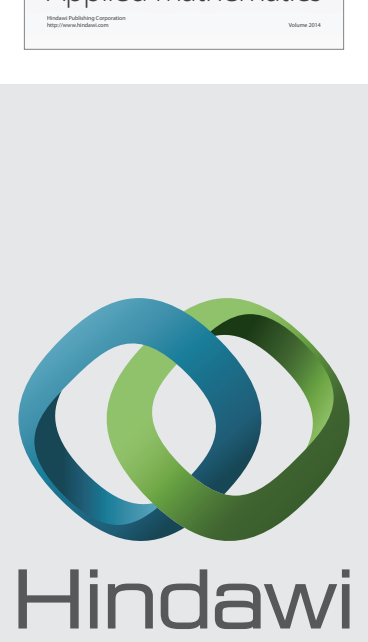

Submit your manuscripts at http://www.hindawi.com
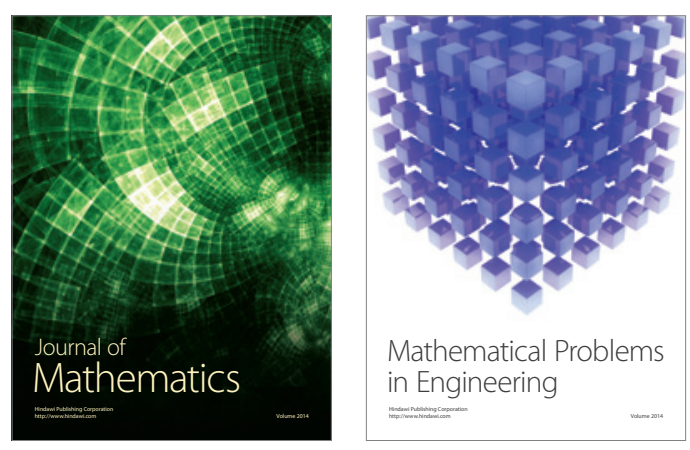

Mathematical Problems in Engineering
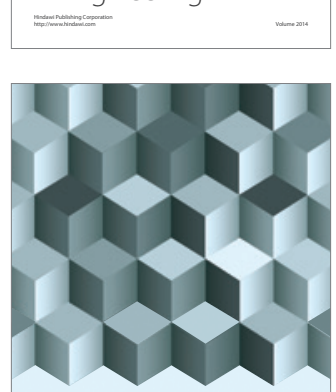

Journal of

Function Spaces
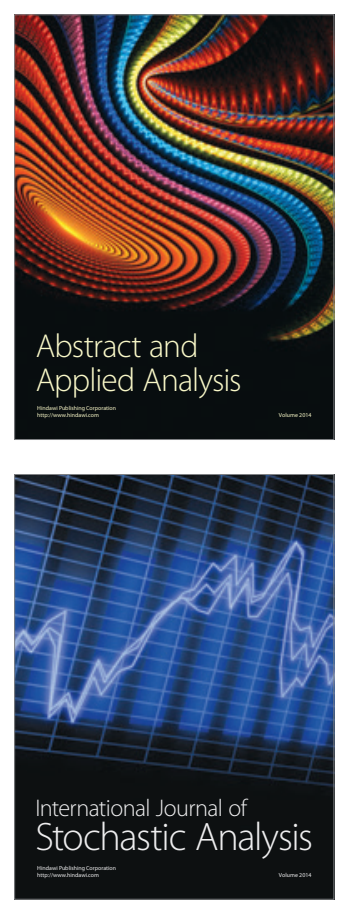

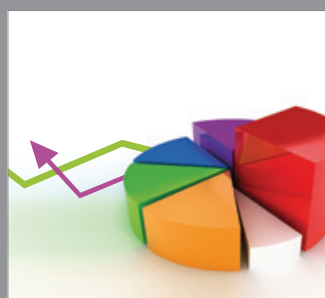

ournal of

Probability and Statistics

Promensencen
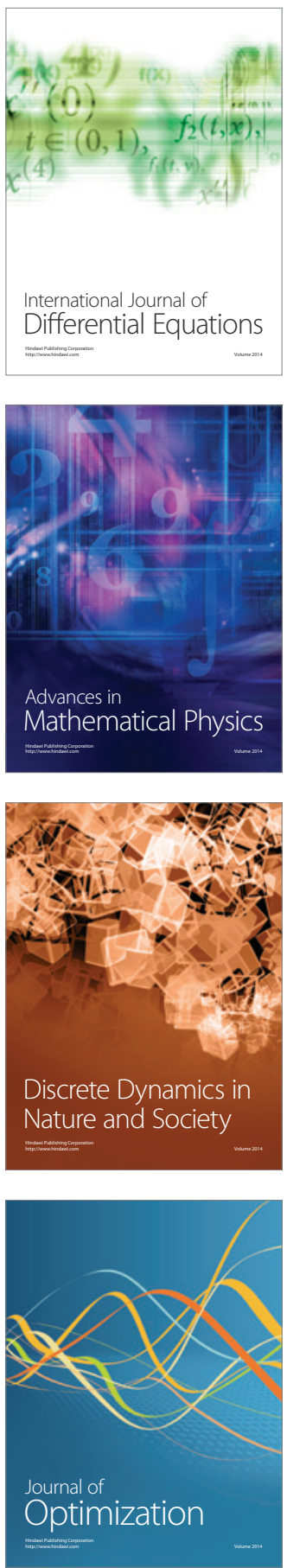